\title{
Application of Dynamic Manufacturing Service Provisioning Mechanism to Delivery Commitment
}

\author{
Yea-Huey Su ${ }^{1}$, Shi-Chung Chang ${ }^{2}$, Ruey-Shan Guo ${ }^{3}$, Yi Chang Lai ${ }^{4}$ \\ ${ }^{1,3}$ Graduate Institute of Business Administration, National Taiwan University \\ ${ }^{2,4}$ Department of Electrical Engineering, National Taiwan University
}

\begin{abstract}
The concept of "virtual fab" emphasizes on manufacturing service provisioning. This paper designs a dynamic manufacturing service provisioning mechanism (DMSPM) for the creation, deployment, and execution of manufacturing services in a virtual fab (VF) environment with quality-of-service (QOS) guarantees. DMSPM exploits object-oriented technology to flexibly bind fab objects into services. To examine our ideas of DMSPM, the order commitment service (OCS) provided by foundry companies serves as a study case. A prototype is designed and implemented for realizing DMSPM in the OCS application by using the unified modeling language (UML) for object oriented modeling and a CASE tool for $\mathrm{C}++$ code development. Results of experimentation with the prototype demonstrate both the ideas and the potential of DMSPM for application to virtual fab and e-business developments.
\end{abstract}

\section{Introduction}

Fast growth of foundry fabs, fierce global competition and advancements of information technology have motivated in the semiconductor industry the concept of "virtual fab"[1][2] with emphasis on manufacturing service provisioning. To facilitate the development of a virtual fab, Su et al [3] have proposed a virtual fab enabling framework and Chang et al [4] have designed a dynamic binding mechanism for manufacturing service creation with guaranteed quality via effective access to all the fab resources.

The dynamic binding mechanism is designed on top of the object-oriented (OO) model of a fab. A computer-aided software engineering (CASE) tool, Rational Rose [9], is adopted as an enabler of the dynamic binding mechanism, which supports a range of functions from $\mathrm{OO}$ model construction to $\mathrm{C}++$ code generation. However, only have simple examples been previously studied to assess the feasibility of these ideas. In this paper, the dynamic binding mechanism is further refined into a dynamic manufacturing service provisioning mechanism (DMSPM). The order commitment service (OCS) provided by foundry companies such as TSMC and UMC is then extracted as a study case to examine our ideas of DMSPM.

The remainder of this paper is organized as follows. In section 2 , ideas about virtual fab and manufacturing service are reviewed. In section 3 the manufacturing service provisioning skeleton is first proposed, followed by the design of dynamic manufacturing services provisioning mechanism (DMSPM). An OCS example is used to demonstrate its potential for real application in section 4 . Finally, section 5 concludes this paper. 


\section{Virtual Fab and Manufacturing Service}

Recently, the idea of "virtual fab (VF)" with manufacturing service has been proposed by the industry as one of the critical aspects for achieving competitiveness[1][2]. The VF idea may conduct a service-oriented new business model to IC industry. The business model of running a foundry fab is totally different from that of a product fab and the value-added activity cannot be analyzed purely based on the traditional "manufacturing" model. A new model that provides customers with " manufacturing service" in addition to manufacturing capability must be in place. Quality, flexible, and quick manufacturing service provisioning is the driving force for the development of VF.

A manufacturing services provider delivers some intangible products in the manufacturing processes or delivers some intangible products together with required tangible products simultaneously to customers. This paper defines manufacturing services as both tangible products and intangible products in which customers involve in part of manufacturing processes. Tangible products are real and touchable entities such as products produced from fab. Intangible products can be classified into three types: serviced time, information, and possession as described in Table 1.

In order to provide manufacturing services, a three-layer enabling framework for VF has been proposed by $\mathrm{Su}$ et al [3]. In the VF enabling framework (Figure 1), objects with different abstraction in a fab are distributed among three layers: manufacturing service layer, business process layer, and infrastructure layer. The top layer, manufacturing service layer, has two functions: presentation and service composition and decomposition. The presentation function takes and interprets service requests from outside partners or customers and delivers manufacturing services to them. Service composition and decomposition function decomposes the requested services into business processes and and binds them from the two lower layers to provide the requested manufacturing service. The business processes (BP) layer composes business activities and resources from the infrastructure layer into a business process. The VF infrastructure layer consists of abstract modeling objects and has four key ingredients: data warehouse, open real-time simulation, DMSPM server, and customer-oriented interface.

The three layers work together on a manufacturing service request but with different functionality. This design of three-layered VF framework facilitates flexible manufacturing services provisioning. Flexibility means easy to add, delete or modify services without changing the framework. To provide flexible manufacturing services, business processes (BP) have to be reusable in different manufacturing services and can be dynamically configured. Addition of new BPs and modification of infrastructure do not occur frequently. Even when addition of new BPs and modification of infrastructure is needed, the effect of changes can be confined locally. The reusability of business process facilitates the dynamic composition of business processes into various manufacturing services.

\section{Dynamic Manufacturing Service Provisioning Mechanism (DMSPM)}

This section proposes a specific way, dynamic manufacturing service provisioning mechanism (DMSPM), for VF framework to flexibly provide manufacturing services. DMSPM exploits the object-oriented methodology, including model abstraction of a fab, analysis, design, and implementation techniques. Consider a virtual fab environment 
built on three-layered VF enabling framework [3] and an object-oriented (OO) fab model [6][7]. DMSPM is designed as follows.

\section{A. Manufacturing Service Provisioning Skeleton}

The process of manufacturing service provisioning in DMSPM can be broken into three phases in sequence: creation, deployment, and execution. First, creation phase plans or designs how a requested manufacturing service should be fulfilled. Second, deployment phase organizes and prepares available resources so that they are ready for immediate action. Finally, execution phase carries out the manufacturing service fulfillment plan in the way that has been created. Although the three phases are executed at different time with different binding concept, they follow common steps: name mapping, business process binding, resource reservation binding, and manufacturing service management binding.

A skeleton of the DMSPM is extracted from the commonality of three phases. Figure 2 depicts this manufacturing service provisioning skeleton. The input is manufacturing service request with quality of service (QOS) requirements from customers and the output is the managed manufacturing service. The DMSPM skeleton consists of four major steps. The first is "name mapping", which converts an external order into internal work orders. The second step, "business process binding," transfers internal work orders into business process flows. The third step, "resource reservation binding," reserves resources such as machine capacities, human resources, and raw materials of the factory. Finally, "service management binding" binds the service plan to make it a managed service.

How the skeleton is applied to the three phases is described below. First, the creation phase consists of four steps:

- Name mapping: Automatically translate an external manufacturing service request into the specification expressed in internally recognizable terms and thereby create an internal service requirement.

- Business processes binding: Associate (bind) related business processes to the internal service requirement and thereby create workflows which are sequences of activities with the requirements of time and quantity to resources.

- Resource reservation binding: Associate required resources to the generated workflows. Then reserve and schedule the use of necessary resources and thereby create a fab service plan. This step integrates functions such as resource registration, activation, scheduling and optimization. Resource registration is to take all resource available into binding service's control umbrella. Resource management is to automate the resource scheduling, optimization and allocation processes.

- Manufacturing service management binding: Associate manufacturing service managers/systems or managerial activities in each layer to the fab service plan to make sure the feasibility of such plan. The activities of management include auditing such as setting up checkpoints, costing, and QOS management.

Similarly, the deployment phase consists of three steps:

- Business processes binding: Activate (bind) the business processes of the feasible fab service plan created in the first phase to be ready for immediate action and thereby the workflows is ready to run. 
- Resource reservation binding: Activate preparation and organization of the planned resources so that they are in a position or condition to action immediately. Thereby the feasible fab service plan created in the first phase is ready for action.

- Manufacturing service management binding: Activate manufacturing service managers/systems in each layer to the feasible fab service plan to make sure this plan is executable as planning.

Finally, execution phase consists of three steps:

- Business processes binding: Activate (bind) the relative business processes to carry out the control of workflows for executable plan.

- Resource reservation binding: Activate planned resources to carry out the manufacturing service fulfillment plan in the way that has been created and deployed.

- Manufacturing service management binding: Activate manufacturing service managers/systems to carry out the managerial activities in each layer and thereby output the managed manufacturing service to customers.

\section{B. Interactions between Skeleton of DMSPM and VF Framework}

Figure 3 illustrates the interactions between steps of skeleton (Figure 2) and the three-layered virtual fab framework [3]. Together they represent the complete view of DMSPM, which illustrates how objects of the three layers are basically bound into a service. A manufacturing service is described with the interactions of various business processes located at business process (BP) layer. The infrastructure (IR) layer contains all the resources needed for the execution of business processes and bound to them at plan time and run time. A customer requests manufacturing service via the interface object in the manufacturing service (MS) layer. After name mapping by such interface object, the related MS objects in MS layer further binds BP objects in BP layer for internal service requirements to generate workflow for this manufacturing service. Those BP objects then bind associated resources in IR layer to create fab service plan in creation time and to carry out in run time. Manufacturing service managers/systems for managerial activities are finally bound out by service related objects in each layer to facilitate the quality management and thereby output the managed manufacturing service to customers.

A service provisioning is "dynamic" in that DMSPM can flexibly provide manufacturing services to meet requirement changes of customers based on the current status feedback of a fab. That is, in response to change of customer requests and fab status, service plan can be quickly created in details about what a fab is going to do and how a fab is going to fulfill the requested manufacturing service. However, the plan does not reserve or assign any particular resources. Only in the deployment and execution phase are particular resources allocated. The characteristics of reusability and contingency for objects are the roots of the flexibility for DMSPM.

How the DMSPM can be enabled by available technologies is now assessed. Database technique that records the objects together with their relationship and behavior is essential for the implementation of DMSPM. To support quickly look-up and mapping, a data-organized technology such as look-up table or catalog technique is required other than database technique. Moreover, for the binding actions for steps in the skeleton, the dynamic linkage technology is necessary. Finally, cost model is also required as one kind of the criteria for both steps of "resource reservation binding" and "manufacturing 
services management binding". In specific, the techniques of run-anywhere web and individual portfolio are the essences for manufacturing service interface. The techniques of bill of material (BOM), customized interpreter, standardized data exchange, and name translator is implemented to fulfill name and resource mapping. The global QOS to local QOS translation technique is required to fulfill business process binding. The capacity constraints require the planning/scheduling engine and QOS to resource requirement translation for the implementation of resource reservation binding. For example, commercial software such as ERP program tool can be used to perform production plan or scheduling to calculate resource quantity and time to be reserved for QOS provisioning. Finally, audit technology which determines the check points with frequency and carries out to track quality, cost, time, and progresses is very important to fulfill manufacturing services management binding and complete the DMSPM.

\section{Case Study: Order Commitment Service (OCS)}

A simplified example of an order commitment service (OCS) is adopted to illustrate DMSPM and to assess its practicality. Order commitment service is an important service in a fab to ensure quick response of committed due date to customers once customers placing orders. Figure 4 depicts an abstracted workflow of OCS, which is extracted from the practices of TSMC and UMC and is also locally called an available-to-promise (ATP) service. As shown in Figure 4, an OCS takes customer orders as inputs and binds information resources of necessary procedures. Then OCS evaluates the capacity situation and calculates due dates by using a resource reservation algorithm. This system finally confirms the due dates with individual customers to complete the OCS. A good OCS must provide customers with quick responses, transparent business processes and credible delivery schedules. The inputs of OCS include an order with expected due date while the output includes the committed due date and the wafer out plan.

Figure 5 indicates the objects abstracted from Figure 4 via object-oriented modeling approach. These objects belong to the three layers of VF framework. The static relationships among objects are predefined. For every manufacturing service, the contact window in MS layer is associated to customer-oriented interface in the IR layer via system initialization $\mathrm{BP}$ in the BP layer. An OCS MS involves both OCS BP and wafer out plan BP which consist of other BPs such as negotiation BP or capacity allocation in BP layer. Resource objects in infrastructure layer are needed to accomplish activities of BPs. For example, sales staff and production-plan staff negotiation carry out the activities in negotiation BP while open real time simulation server fulfills both activities of residual capacity calculation and capacity requirement calculation in BP layer.

In Figure 6, the detailed processes follow the common steps of DMSPM skeleton for creation, deployment, and execution phases for OCS provisioning. In the creation phase, customer object requests OCS by sending messages of order specification to contact window object in MS layer. By name mapping, contact window object then translates this OCS request into internal service requirements which are sent to OCS MS object in MS layer. This OCS MS object further binds associated business process objects by using its operation of business process binding. By reservation binding, business process objects then bind necessary resource objects and reserve them in the infrastructure layer to 
form a service plan. The manager objects are bound to check whether the service plan is feasible. After receiving positive response from manager objects, those resource objects send message to their associate BP objects to ensure this plan is feasible through managers' confirmation. Similarly, those BP objects and OCS MS object follow the same idea and processes to carry out the step of manufacturing service management binding and the creation phase is then completed. The deployment and execution phases follow the same idea and steps from business process binding to manufacturing service management binding but emphasize on objects and service activation.

Consider an example of OCS service provisioning process in a foundry XYZ, where customer NTU who requires confirmation for due date at day 20 orders 235 wafers of DRAM with $0.25 \mu$ technology at day $111: 00 \mathrm{am}$. Figure 7 indicates the step of name mapping for OCS. As shown in Figure 8, the OCS MS object in MS layer then sends internal OCS MS requirements to OCS BP object and sends internal MS specifications to wafer out plan BP object in BP layer. These two BP objects then generate the OCS workflows. In resource reservation binding step, workflows then further consider the availability and schedules of associated resources. As illustrated in Figure 9, associated logical and physical resource objects in infrastructure layer are bound by BP objects in BP layer in order to arrange resource reservation plan. Figure 10 illustrates the step of manufacturing service management binding for those objects mention above distributed in three layers to bind their own manger objects.

Ideas of the DMSPM with application to OCS have been proposed and illustrated above. Conceptually, the mechanism is service driven and existing OO-based commercial CASE tools such as Rational Rose [9] can be used to realize it. A CASE tool first allows us to define user cases that describe the client-server relationship between objects. Classes of objects are then pictorially defined. Message passing or interaction sequence diagram can be easily generated from the object model. A CASE tool may also support a code generation function, which yields a code framework of the $\mathrm{OO}$ model built. The process of service provisioning naturally falls into a structure suitable for activity-based costing [8] and QOS requirement derivations.

A prototype for realizing DMSPM in the OCS application is designed and implemented. In our prototype development, it is found that input data and/or information to DMSPM are mostly available from the information system of a fab and its company. For example, because business processes documents are available via ISO 9000 verification, input data and/or information are essentially available but need to be computerized for those fabs passing ISO 9000 series of qualifications. Figure 11 illustrates the manufacturing service provisioning system architecture. Figure 12 then specifies the DMSPM server architecture for OCS. Based on an OCS model expressed in a unified modeling language (UML) format [9][10], the C++ code implementation of the DMSPM server exploits the code skeleton generated by the CASE tool. In specific, without resorting to a commercial package, a resource reservation algorithm is designed and coded in Java [11]. However, there is no difficulty to replace it with a commercial package. Figure 13 shows the input and output of OCS in a browser screen to customers. Our experimentation results demonstrate both the ideas and the potential of DMSPM for application to virtual fab and e-business developments. 


\section{Conclusions}

In this paper, a dynamic manufacturing service provisioning mechanism (DMSPM) has been designed for the creation, deployment, and execution of manufacturing services in a virtual fab (VF). DMSPM is based on the object-oriented methodology to flexibly bind fab objects into services. The order commitment service (OCS) provided by foundry companies has served as a study case to examine the ideas of DMSPM. A prototype has been designed and implemented for realizing DMSPM in the OCS application by using the unified modeling language (UML) for object oriented modeling and a CASE tool for $\mathrm{C}++$ code development. Results have demonstrated that the ideas are feasible under current fab practice of information technology. DMSPM should have a good potential in application to virtual fab and e-business developments.

Table 1 Characteristics of tangible products and three types of intangible products

\begin{tabular}{|c|c|l|}
\hline \multicolumn{2}{|c|}{ Product Type } & \multicolumn{1}{c|}{ Characteristics } \\
\hline \multirow{4}{*}{$\begin{array}{c}\text { Intangible } \\
\text { Products }\end{array}$} & Serviced time & $\begin{array}{l}\text { - Periods available for specific purposes } \\
\text { - Customers consume the time of professional staffs }\end{array}$ \\
\cline { 2 - 3 } & Information & $\begin{array}{l}\text { - Knowledge of know-how, or data of facts/status/capability } \\
\text { for a specific system (e.g. a fab) } \\
\text { - Customers acquire their concerned knowledge or data }\end{array}$ \\
\hline \multicolumn{2}{|c|}{ Tangible Products } & $\begin{array}{l}\text { - Token or reservation right from fab during a specific period } \\
\text { - Customers keep the token or right for exchange related } \\
\text { service(s) in specific conditions }\end{array}$ \\
\hline
\end{tabular}

\begin{tabular}{|c|}
\hline Manufacturing Service Layer \\
\hline Business Process Layer \\
\hline Infrastructure Layer \\
\hline
\end{tabular}

Figure 1 Three-layered VF framework

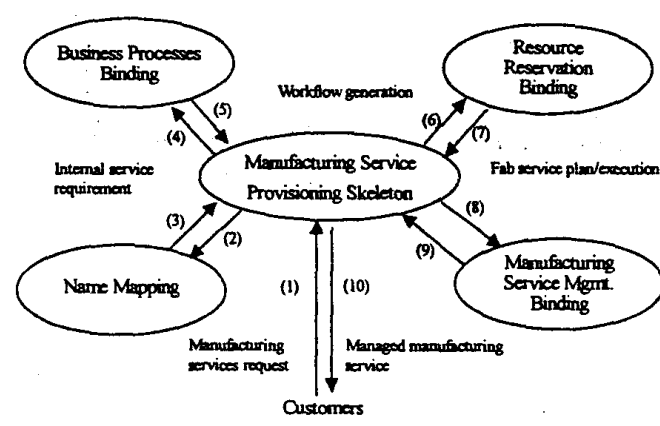

Figure 2 Manufacturing service provisioning skeleton

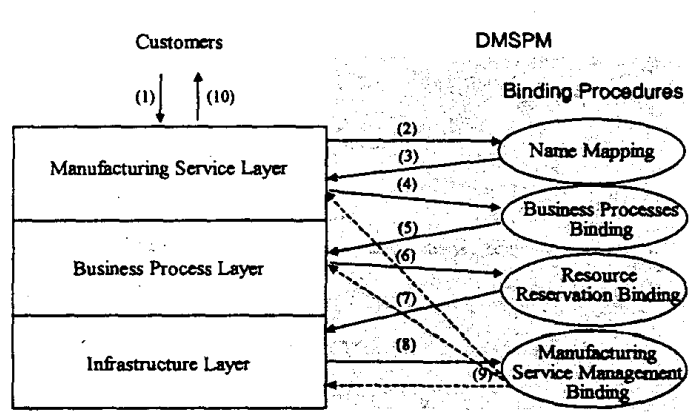

Figure 3 Interactions between skeleton of DMSPM and VF framework 


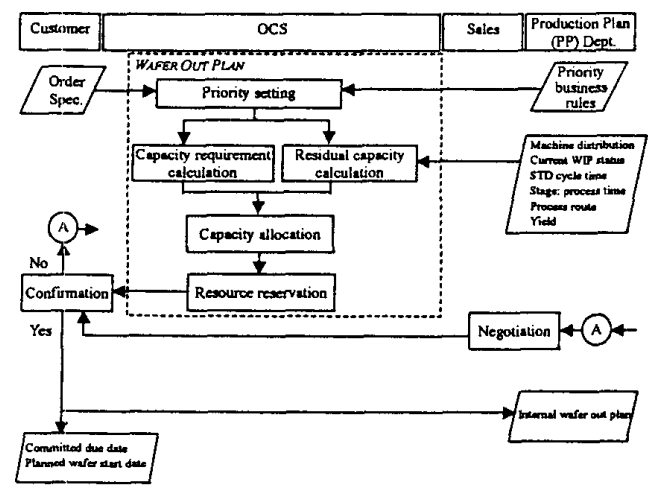

Figure 4 Order commitment service (OCS) workflow
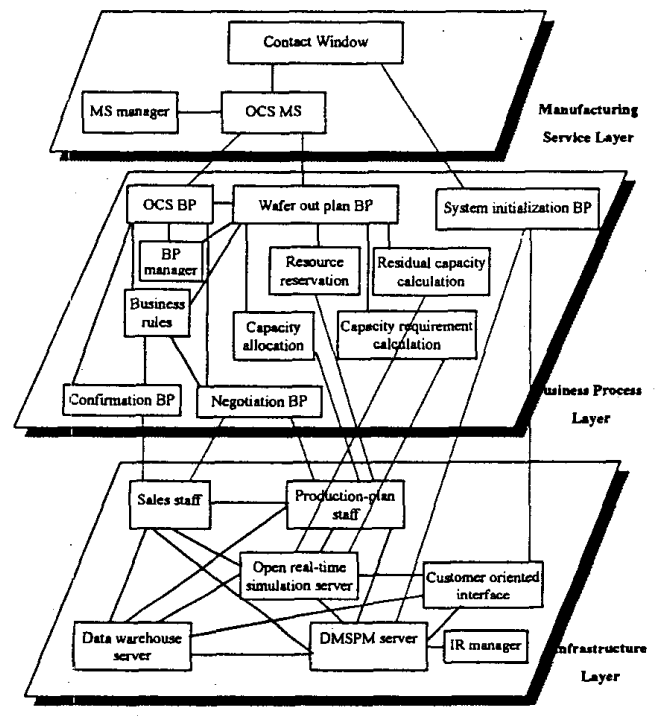

Figure 5 The static relationship of objects distributed on three layers for OCS provisioning

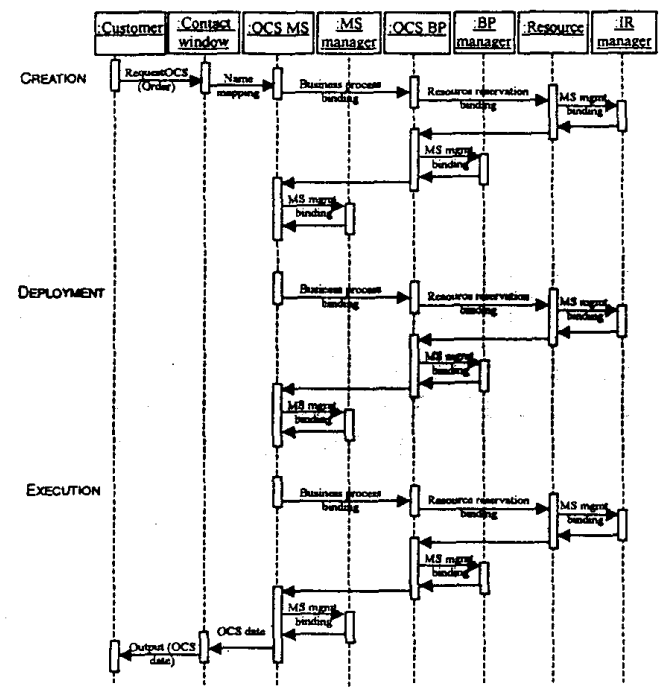

Figure 6 Sequences of OCS manufacturing service provisioning in DMSPM

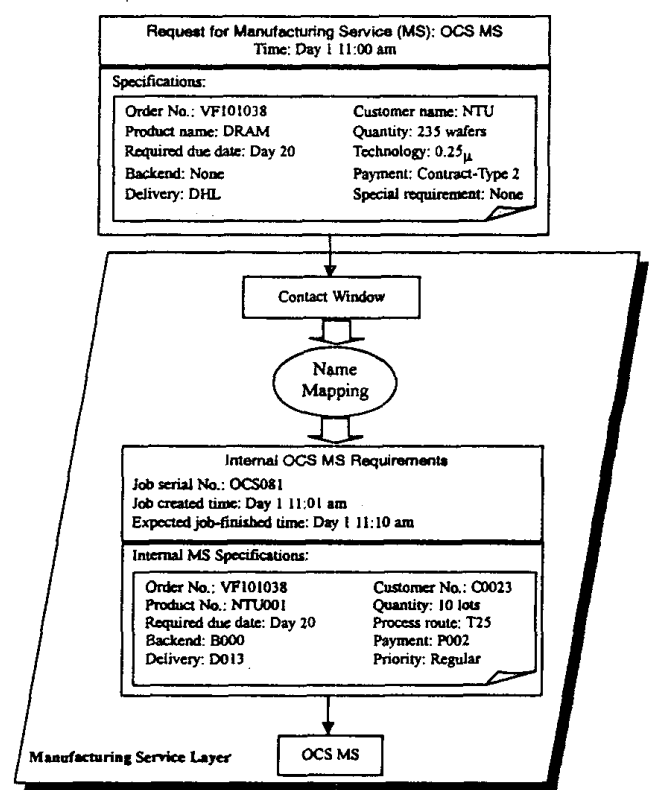

Figure 7 Name mapping for OCS 


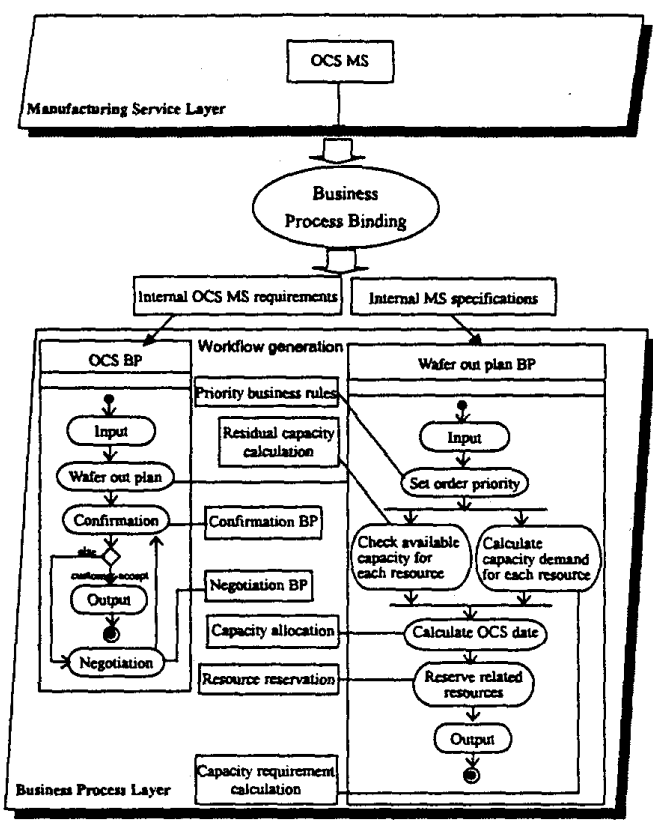

Figure 8 Business process binding for OCS

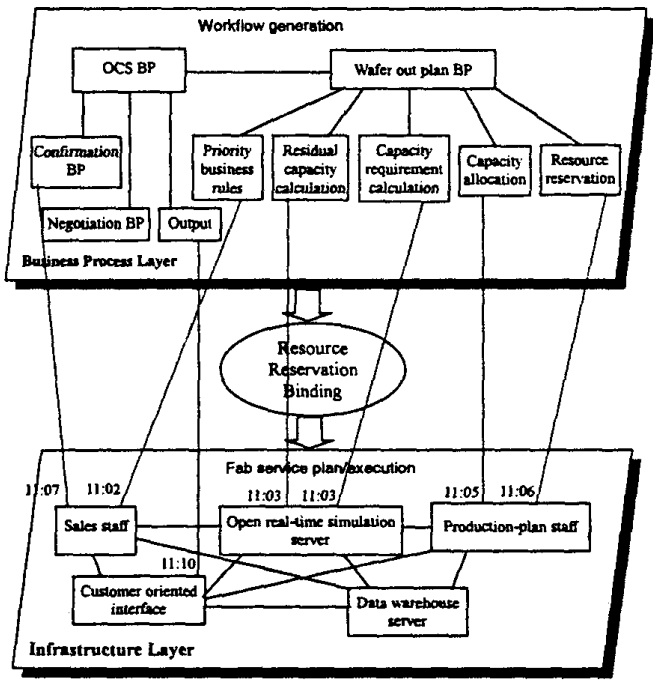

Figure 9 Resource reservation binding for OCS

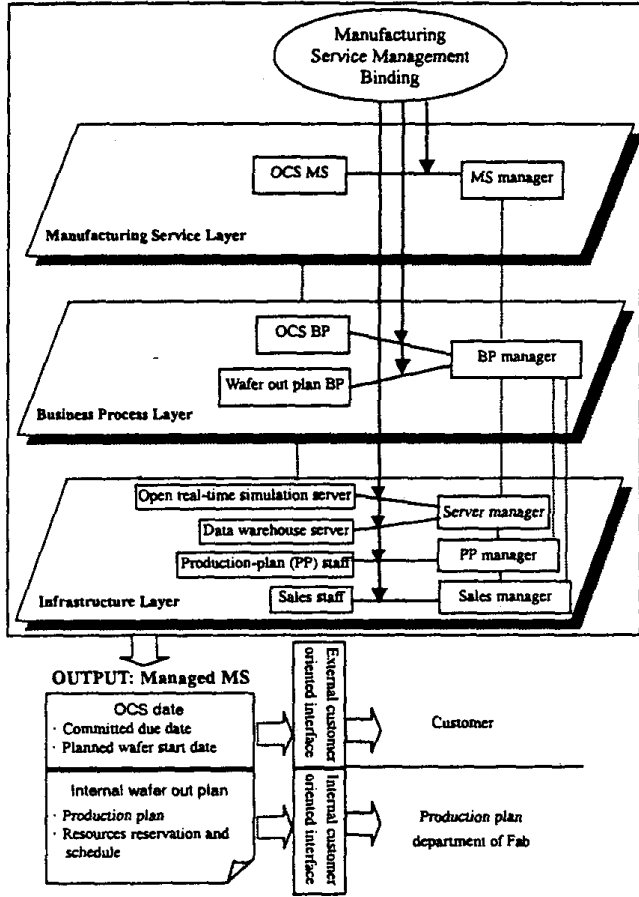

Figure 10 Manufacturing service management binding for OCS

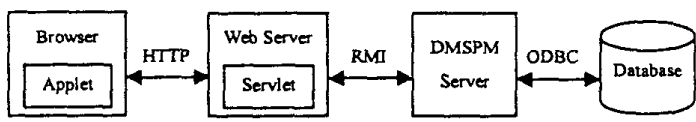

Figure 11 Manufacturing service provisioning system architecture

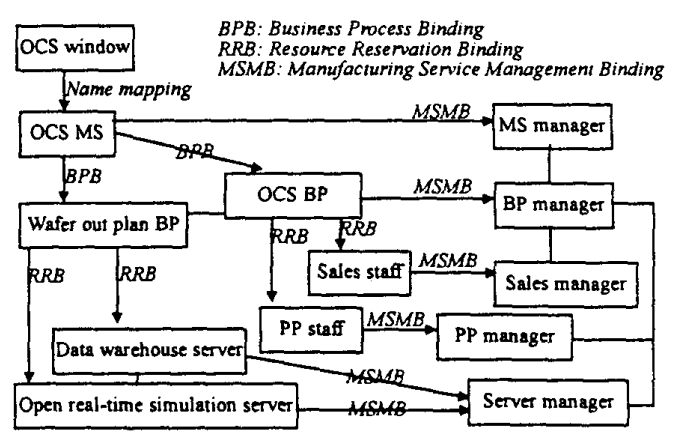

Figure 12 DMSPM server architecture for OCS 


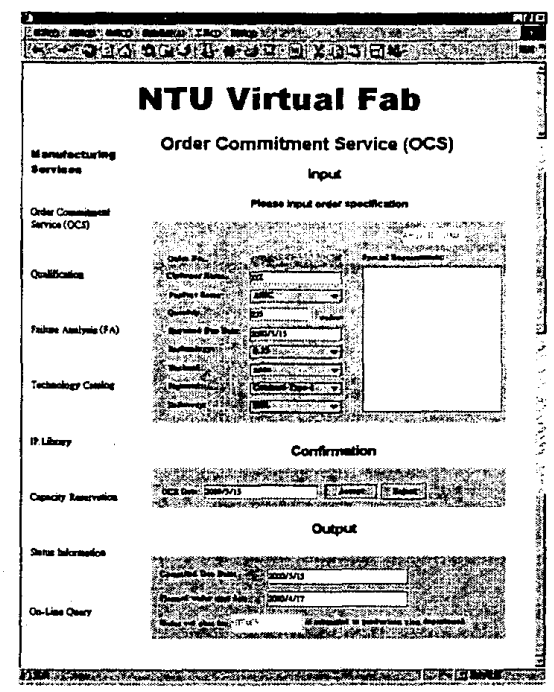

Figure 13 Input/output of OCS in browser screen 


\section{References}

[1] Semiconductor Industry Association (SIA), The National Technology Roadmap For Semiconductor, San Jose: SIA, 1994.

[2] F. C. Tseng, "TSMC's semiconductor strategy (invited)," Keynote speech of Proceedings of the Seventh International Symposium on Semiconductor Manufacturing, pp. 5-7, Tokyo Japan, October 7 9, 1998.

[3] Y. H. Su, R. S. Guo, S. C. Chang, T. L. Chou, Y. C. Lai, "Manufacturing Service Creation and Management for Next Generation Virtual Fab," Proceedings of International Symposium on Semiconductor Manufacturing 1998, Tokyo, Oct. 7-9, 1998, pp. 27 30.

[4] S. C. Chang, T. L. Chou, R. S. Guo, Y. H. Su, L. L. Lu, "A Dynamic Binding Model for Service Creation in Virtual fab," Proceedings of 1998 Semiconductor Manufacturing Technology Workshop, Hsinchu Taiwan, June 16-17, 1998, pp. 131-137.

[5] A. A. Lazar, K.S. Lim, and F. Marconcini, "Realizing a foundation for programmability of ATM networks with the binding architecture," IEEE Journal on Selected Areas in Communications, vol.14, no.7, pp. 1214-1227.

[6] ESPRIT/AMICE, CIMOSA: Open System Architecture for CIM, Berlin: Springer-Verlag, 1993.

[7] J. McGehee, J. Hebley, and J. Mahaffey, "The MMST computer-integrated manufacturing system framework," IEEE Transactions on Semiconductor Manufacturing, vol.7, no.2, pp. 107-115, May 1994.

[8] R. W. Hilton, "Ch5 Activity-Based Costing (ABC) and Cost Management Systems; Ch6 Activity-Based Management (ABM) and the New Manufacturing Environment," Managerial Accounting, New York: McGraw-Hill, 1997.

[9] Quatrani, Visual Modeling with Rational Rose and UML, Addison-Wesley Publishing Company, 1998.

[10] J. Rumbaugh, I. Jacobson, and G. Booch, The Unified Modeling Language Reference Manual, Addison-Wesley, 1999.

[11]Bruce Eckel, Thinking in Java (2 ${ }^{\text {nd }}$ ed.), President, MindView, Inc., 2000. 